\title{
Combined Analysis of Local Influences and Multipath Effects for a Lane-Specific Positioning of Vehicles in Traffic Streams
}

\author{
Brian Niehoefer, Sarah Lehnhausen, Christian Wietfeld \\ Communication Networks Institute \\ TU Dortmund University \\ Dortmund, Germany \\ brian.niehoefer|sarah.lehnhausen|christian.wietfeld@tu-dortmund.de
}

\begin{abstract}
Future smart traffic environments require very accurate positioning of vehicles to allow for sophisticated traffic flow modeling or autonomous control of cars. To avoid traffic congestion on motorways, a lane-specific localization of vehicles is required to allow for the detection of short-term deviations of traffic flows due to roadwork or accidents. In this paper, we investigate the accuracy of GNSS-based positioning data with consideration of all relevant impairments of the propagation channel, like molecular scattering, ionospheric scintillation and effects caused by the direct receiver surrounding. Taking into account satellite mobility and constellations, position-specific atmospheric influences and the impacts of shadowing and multipath propagation on the determination of TOA values, the accuracy of a lane-specific positioning is investigated for both GPS and GALILEO. The results presented in this paper are based on a dedicated multiscale simulation framework which includes an exact 3D model of the environment of the GNSS receiver. Based on the investigation of a reference scenario, the results demonstrate the feasibility of lane-specific localization enabled by the compensation of both ionospheric as well as multipath fading effects.
\end{abstract}

\section{INTRODUCTION}

The accuracy of satellite positioning systems like GPS, Glonass or the future Galileo are increasing continuously, whereby applications in areas like navigation, location-based services and logistics management are the main beneficiaries of these technological improvements. But, despite all progress in satellite and localization technology, there are still many elements of uncertainty in evaluating the satellite positioning accuracy for a specific point on earth at a specific time. The direct receiver's surrounding or ionospheric effects are just two examples which highly affect the positioning precision. Applications using satellite positioning systems are limited to services which do not rely on a permanently guaranteed localization accuracy. One possibility to include the influences is empirical or semi-empirical wave-propagation, whereby many occurring phenomenas are already gathered in analytical models. But those are mostly based on real world measurements in a specific environment and thus, not generally valid.

To overcome this limitation and to enhance future applications, the Communication Networks Institute has developed, in the context of the Collaborative Research Project 876 of the
German Research Foundation, a highly accurate simulation model which allows to determine the localization accuracy using raytracing technology and ionospheric considerations for a given satellite constellation at a random position on earth. The framework includes all influences of the ionosphere as well as the receiver's surrounding which have a non-negligible effect to the localization signal. Thereby separated tools, like protocol simulator, raytracer and 3D visualizing tools are combined with heterogeneous data sources like weather or model databases and EGNOS data (via SISNeT) within a multiscale simulation framework. To clarify the benefit, the gained additional information is used to enhance 'lanespecific' traffic-flow predictions using microscopic traffic models, like the Nagel-Schreckenberg Model [1]. This enhancement would have a crucial impact on traffic forecasts and/or -news considering daily fluctuations in all traffic networks, due to accidents or road works.

In this contribution the authors will first explain the benefit of lane-specific positioning methods in Section II. Afterwards, the underlying motivation of using a combined framework including raytracing for this kind of scientific challenge as well as similar approaches will be discussed in Section III, followed by the multiscale simulation modeling approach in Section IV, which has been previously used in the context of the evaluation of multipath-effects to satellite-based positioning systems and which is now extended by new functionalities within the so-called Simulative Positioning Accuracy Determination (SPAD). The results in Section V include a detailed discussion of the evaluation of the lane-specific positioning accuracy of different satellite systems and the possible accuracy enhancement using an integration of all local, atmospheric and multipath effects with the developed framework. A conclusions and an outlook wrap up the paper at the end.

\section{ImPaCt of LANe-SPECific Positioning Data}

The prognosis of autonomous objects moving in streams within a given complex street network, e.g. automobile traffic or people crowd flows, is a challenging scientific question. Given an accurate prognosis, an optimal occupancy rate on the respectively used graph can be enabled by guiding individual 
objects on alternative routes, if necessary. The accuracy of the acquired data and a minimum delay between data aggregation and stream prediction are essential to assure a useful outcome. In this case, traffic prognosis is used as an example for object flow optimization, with the aim to detect local traffic flow impacts caused by car breakdowns or road work. Research using microscopic modeling of traffic flows has shown, that major traffic jams can be caused by minor disruptions of the traffic flow [2]. The objective is to detect those minor incidents reliably, within a short period of time and with a high precision. Especially the last task can be summarized as one major challenge, the lane-specific detection of incidents on a continuous basis. Current traffic-flow prediction methods typically rely on discrete measurement points, like induction loops or infrared/video systems [3]. Assuming the use of obstacles (vehicles) as a kind of intra traffic-flow sensor nodes, it is possible to detect any manipulation of the traffic flow even between those discrete measurement points.

Nevertheless, every satellite positioning system has certain inaccuracies like time bias failures [1], ionospheric or multipath effects [4][5] that make it difficult to guarantee the necessary accuracy all over the time and location-independent. Figure 1 shows an exemplary visualizations of different GPS tracks (dashed lines) and the real driven route (solid line) depending on the respectively traveled distance $s$.

The error concerning the lane-specific positioning is called $\Delta x$. Assuming a $\Delta x$ of zero, every measured position $\left(\vec{x}_{G P S}\right)$ would be within the boundaries $b_{z}$ of the given lane (cmp. to Figure $1 \mathrm{z}=1,2,3)$, but not inevitably equal to the real position $\left(\vec{x}_{\text {real }}\right)$ according to the following formulas [6]:

$$
\begin{array}{r}
\vec{x}_{i} \in \vec{b}_{z}\left|\min _{s, z}\right| \vec{x}_{\text {real }}-\vec{b}_{z}(s) \mid \\
\vec{x}_{j} \in \vec{b}_{z}\left|\min _{s, z \neq i}\right| \vec{x}_{r e a l}-\vec{b}_{z}(s) \mid \\
\Delta x=\min \left(\min _{s}\left|\vec{x}_{G P S}-\vec{x}_{i}(s)\right|, \min _{s}\left|\vec{x}_{G P S}-\vec{x}_{j}(s)\right|\right)
\end{array}
$$

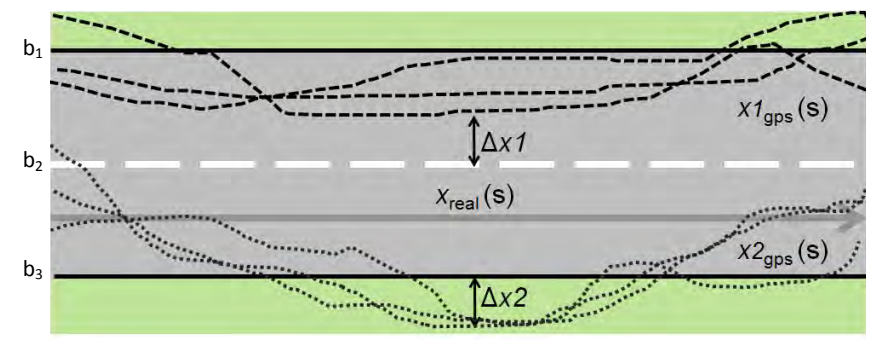

Fig. 1. Clarification of 'lane-specific' positioning

Assuming a compensation of this unpreventable effects to satellite positioning signals, the full benefit of this data aggregation mechanism becomes visible. Using this approach for a time-continuous aggregation of lane-specific positioning data would enable a reliable detection of temporary deviations within the traffic flow and a cost-efficient method to gather the lane-specific positioning data.

\section{Accuracy Estimations for Satellite-Based Positioning - MECHANISMS AND RESTRICTIONS}

To clarify the potential benefit of using raytracing technology within highly dynamic communication scenarios, this section provides a short state-of-the-art summary of different evaluation techniques for satellite-based positioning systems. Figure 2 visualizes some of these restrictions by providing some measurement results for a single L-band satellite signal at $21^{\circ}$ elevation with different direct receiver surroundings [7][8].

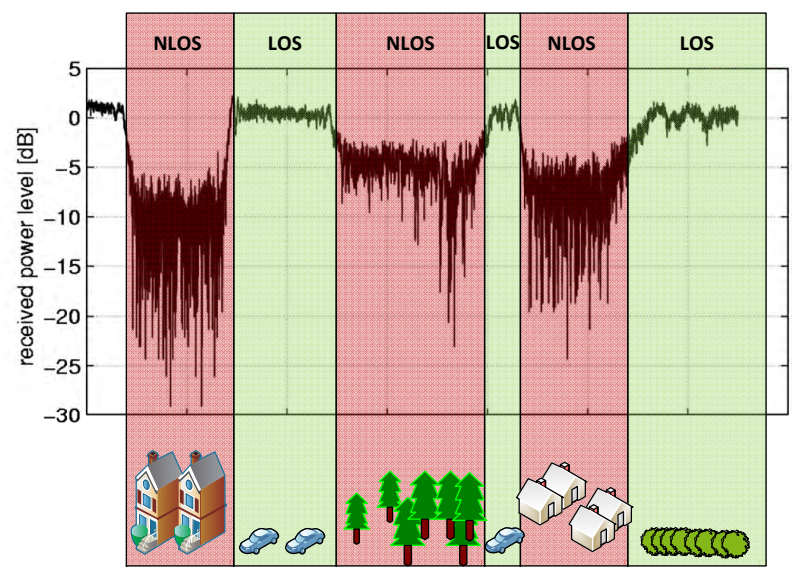

Fig. 2. Measured power level for a land mobile receiver

Based on these measurements, it is obvious that the direct receiver surrounding directly influences the receiving strength and according to multipath effects, also the propagation delay. Even in suburban scenarios without tower buildings and with a moderate building density, there are atmospheric and local influences detectable, which have to be included within a simulative evaluation of satellite signals. Therefore, the restrictions of actual-used models as well as the benefit in using raytracing technology will be highlighted in the upcoming paragraphs.

\section{A. Intrasystem Evaluation - Safety of Life Services}

The Safety-of-Life Service (SoL) improves the open service performance of future satellite positioning systems like Galileo through the provision of timely warnings to the user when it fails to meet certain margins of accuracy (integrity). It is envisaged that a service guarantee will be provided for this service. Mainly designed to support critical applications (avionic), several ground stations are measuring the GPS signal and detect potential deviations. Although there are several stations in operation, a quantification of a specific failure for a specific point on earth is impossible. The SoL is designed to detect the deviation, not to quantify it.

\section{B. Positioning Failure Compensation using additional Sensor Data}

Another research topic since 2011 is the integration of additional sensor data to compensate satellite-based positioning failures, especially for traffic situations. In [9] the fusion of two types of data for the purpose of real-time traffic fusion and 
prediction is considered: GPS data that is provided as point speeds rather than trajectories, as well as (non real-time) traffic data which is available from fixed sensors. Again this approach is based on stationary sensors, which are useless in between. In contrast [10] uses the sensor data from cars, and the exchange of those within the communication range of $\mathrm{WiFi}$, to allow a kind of pre-accident alarm. But again, this data is not used to compensate positioning failures.

\section{Empiric and Semi-Empirical Evaluation}

One possibility to analyze a given radio communication scenario would be the use of analytical models which include many occurring propagation phenomenas. But those models are based on real world measurements in a specific environment. Thus, empirical or semi-empirical wave-propagations are not generally valid [11] and the occurring failure highly depends on the local circumstances in the given situation, e.g. the electronic density and the satellite constellation which both have a significant influence [12]. This was also proven in former publications by comparing empirical as well as raytracing results with experimental measurements using a DVB-S2 Internet-over-Satellite equipment [1].

\section{Position-Specific Evaluation of Communication Aspects using Raytracing Technology}

Simulative radio wave propagations are complex in comparison to alternative methods and their prediction accuracy is always depending on the used modeling level of detail. Raytracing is a well known method in the scientific world to analyze or evaluate random radio-communication scenarios. It can be used to simulate either direct communication aspects (e.g.: path loss models for different technologies like Car2-Car [13]) or indirect but related aspects like localization accuracy, cmp. [6] or [14].

The combination of raytracing technology with tools for simulating satellite mobility, corresponding protocol stacks and an appropriate model database is able to fulfill the requirements mentioned above. The so-called Multiscale Simulation Environment [15] already comes along with those functionalities. Originally designed to analyze and predict the signal strength of local WLAN scenarios, it was adapted to cover also performance evaluations of satellite positioning systems by analyzing signal strength and impulse response of the satellite signals at a specific point on earth. The original setup was already published in [6] and is enhanced by including positionspecific atmospheric effects in this contribution, due to the given scenario-based scientific challenge.

\section{COMbined AnAlysis of LOCAL AND ATMOSPHeriC EFFECTS}

Global Navigation Satellite Systems (GNSS) signals will always be affected by different impacts, decreasing their possible positioning accuracy. As explained in Section III, there are many different ideas to minimize those influences. In contrast, the developed approach in this contribution accepts certain remaining failures and tries to compensate them by using the derived position-specific simulation knowledge for a kind of differential data. This data is called Position-Continuous Differential GNSS Data and will be explained in the upcoming paragraphs.

\section{A. Necessity of Position-Continuous Differential Data}

As mentioned before, satellite systems are highly affected by different atmospheric and local influences. Figure 4 clarifies their dynamic impact with two exemplary visualizations of GPS positioning errors of two consecutive days.

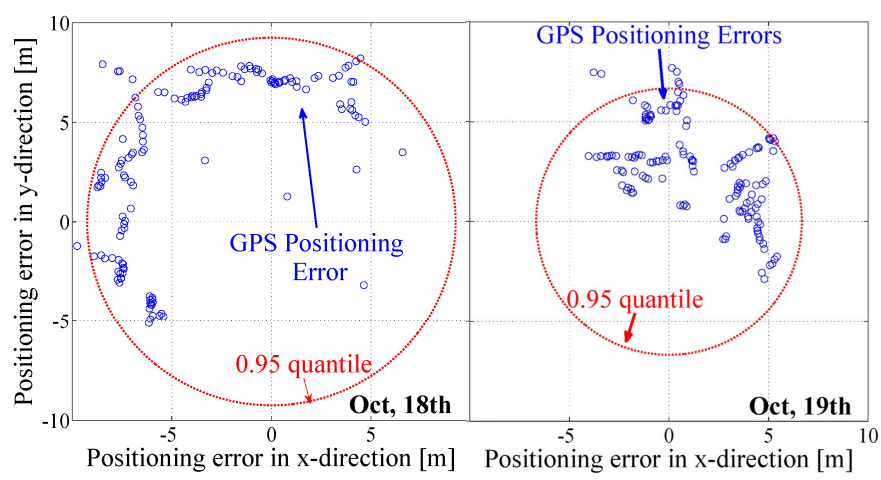

Fig. 4. Daily changes of the Ionospheric Impact to Satellite-based Positioning Systems

Using up-to-date Two-Line Element and EGNOS datasets for each day, the occurring positioning failures for one specific position in the given test scenario was simulated every five minutes for the same 10 hours each day. It is obvious, that the changes in the atmosphere, e.g. the Total Electron Content (TEC), and the relative satellites to receiver constellation is a highly dynamic system and by that underlines the necessity of simulating all occurring effects for each specific time and location without the use of statistical models.

\section{B. Simulation Architecture for GNSS Performance Evaluation}

Based on the idea that it is not suitable to use one tool to cover all aspects of a complex simulation the Multiscale Simulation Environment (MSE) mentioned in Section III-D is used for this kind of scientific challenge. In addition to a discrete event simulator (DES), which is responsible for managing and controlling the simulation tool-chain, a special raytracing tool as well as a highly realistic channel modeling including ionospheric effects is used to gain more detailed results. The communication protocol as well as the geospatial positions of the transmitter and receivers are simulated within the DES. Their respective movement using the appropriate Two-Line-Elements sets of the satellites and the atmospheric effects corresponding to the given satellite-receiver constellation is also included. Without a MSE extension, the calculation of path loss, influences of the weather, ionosphere and receiver's environment could only rely on statistical models and descriptions. In fact, this would reduce the usability of the results.

Figure 3 visualizes the overall architecture of the developed MSE enhancement, called Simulative Positioning Accuracy 


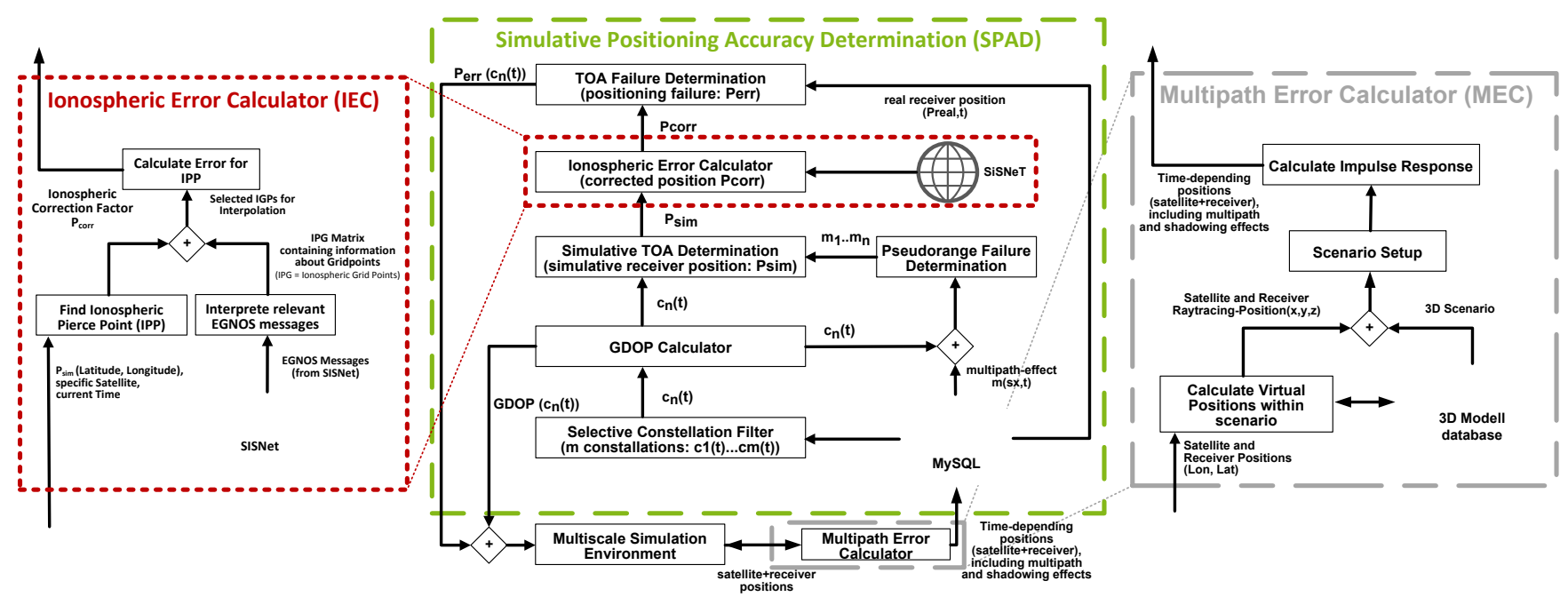

Fig. 3. Satellite Positioning Accuracy Determination (middle) and Functionality of Multipath (right) and Ionospheric Error Determination in Detail (left)

Determination (SPAD) (middle) and the two latest extension, the Ionosperic (left) and the Multipath (right) Error Calculator in more detail. Based on the simulated movement of all satellites and receivers within the MSE, a so-called Selective Constellation Filter (SCF) creates all possible satellite constellations in range. Afterwards every single constellation is analyzed to extract the optimal satellite geometry at a certain time and a given receiver position using the GDOP as one additional key performance indicator. The Multipath Error Calculator (MEC) in Figure 3 right uses the simulated positions of every single satellite in range and the actual receiver to extract the corresponding scenario from a 3D model database. At the moment, this database is limited the university campus $\left(51.4925^{\circ} \mathrm{N}\right.$ and $\left.7.4128^{\circ} \mathrm{E}\right)$, but therefore includes all necessary parameters to set up a highly realistic raytracing scenario, like material properties or geo-referenced models of all buildings. Afterwards, the given positions of the the actual receiver and satellite has to be converted to the Cartesian coordinates of the raytracer, called virtual positions. Using the extracted scenario and the recalculated position of the considered satellite-receiver constellation, the impulse response can be calculated using the given raytracer features. The idea is to use the strongest received signal multipath component for the positioning as long as it is still detectable $(>-189 \mathrm{~dB})$. To calculate the multipath effect, the framework uses the additional delay of the strongest multipath component, compared to the LOS propagation delay. This routine has to be performed for every satellite-receiver combination at a given time, which leads to a resource-intensive calculation. The multipath effect, as well as the underlaying satellite-receiver positions are stored in a database, to enable a pseudorange calculation later on in the SPAD.

Afterwards, the determined position of the receiver as well as the original satellite positions are used, to calculate the non-negligible ionospheric effects (cmp. Figure 3 IEC). Specifically, EGNOS data [16] depending on the used system- time is integrated in the calculation using a SISNeT gateway within Matlab. Based on the time and the simulated position of the object and the satellites, a function calculates the Ionospheric Pierce Point (IPP). In parallel, the latest EGNOS messages are downloaded from SISNeT to gain actual values for the Ionospheric Grid Points (IGPs). Afterwards, the nearest IGPs are extracted and their values are interpolated to reach a realistic statement for the calculated IPP. As a last step, the corresponding error for the given satellite/earth-receiver constellation is returned to the SPAD.

Using all simulated influences, ionospheric as well as multipath effects, the well-known Time-of-Arrival positioning routines are used to calculate the receivers position. The deviation of the real position within the simulation and the calculated one can be interpreted as Onside- and Time-specific Positioning Failure and is returned with the corresponding and derived GDOP value, both depending on the used constellation to the MSE for visualization and storage. Using this knowledge for a specific position and time, an error compensation via post-processing becomes possible.

\section{Accuracy Enhancement using Simulation KNOWLEDGE}

\section{A. Exemplary Urban Scenario}

As mentioned in Section IV-B the framework requires a detailed 3D model database to perform realistic evaluations of all influences on the satellite positioning signals. Hence, an already existing model of the campus of the Technical University in Dortmund is used to analyze the possible performance of the developed ideas.

Figure 5(a) shows a screenshot of the used model, as well as the 25 different Fixed Simulation Points (FSPs) for the analysis. The main pathway of the university is used, which is $6 \mathrm{~m}$ to $11 \mathrm{~m}$ wide and more than $250 \mathrm{~m}$ long, as a kind of virtual two-lane carriageway. The first ten FSPs are surrounded by high buildings, which expectedly results in a high amount of multipath effects. In contrast, the other FSPs should be 


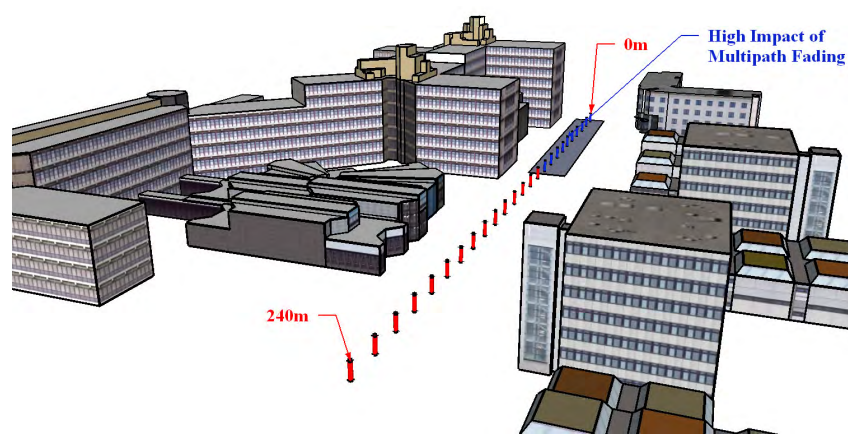

(a) Scenario and used Fixed Simulation Points

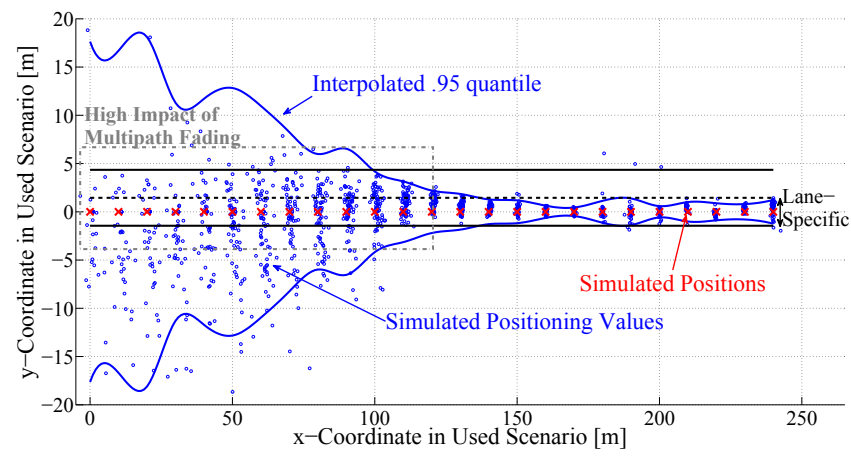

(c) Ionospheric Compensation

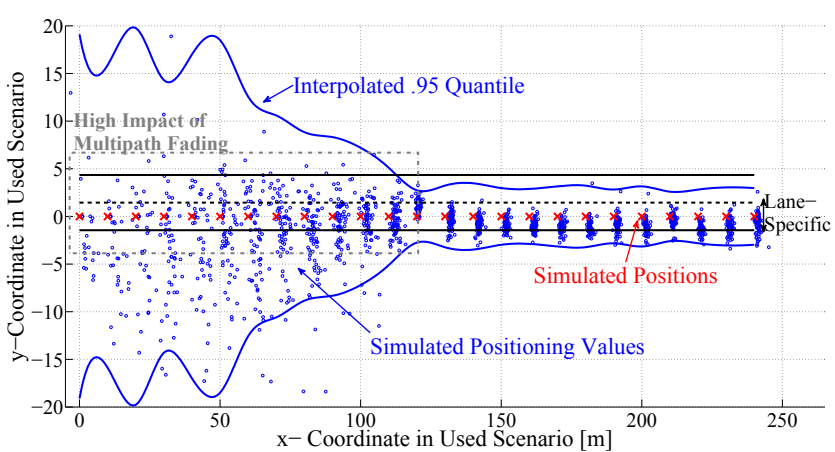

(b) Raw Positioning Data

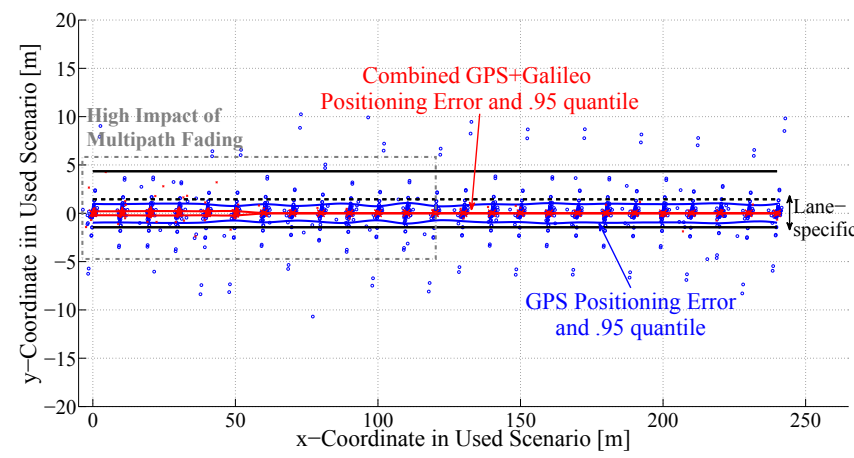

(d) Full Compensation

Fig. 5. Used 3D scenario and Simulated Positioning Data using Different Compensation Techniques

able to receive at least some LOS signals, resulting in a minor multipath effect.

\section{B. Accuracy Evaluation for Lane-Specific Positioning in Traf- fic Applications}

In the following, the authors use all 25 FSPs results in one plot in combination with a 'normal' lane-width of $2.9 \mathrm{~m}$ for a highly descriptive visualization of the possible performance gain using the developed post-processing compensation. Therefore, three different simulative compensation methods are analyzed: Raw GNSS Data (GPS and a combination of GPS and Galileo), Raw GNSS Data with Ionospheric Compensation and Raw GNSS Data with Ionospheric and Multipath Compensation, what is called 'full compensation' in Figure 5(d).

1) Overall Accuracy Determination: Figure 5(b) shows the simulated positioning values and their interpolated .95 quantiles for a combined GPS and Galileo constellation in the test scenario. In addition, all 25 FSPs are marked with crosses in the bottom lane of the marked virtual two-lane carriageway.

As expected, a high amount of multipath impact can be observed for the first working points, due to the high buildings next to the pathway. It is clearly recognizable, that a lane-specific localization is impossible, using this raw data, although 'street-specific' is already reachable for the FSPs 13 to 25 in $90 \%$ of all simulated cases. By eliminating the ionospheric effect in Figure 5(c), some points are already 'lane-specific' in most of all analyzed cases, but the occurring multipath effect still prevents a comprehensive localization of the used FSPs with an error threshold below $1.45 \mathrm{~m}$.

Analyzing the figures in more detail clarifies the multipath impact. Just as an example, the FSPs 7 and 12 are just $50 \mathrm{~m}$ apart from each other, whereby the .95 -quantiles are differing by a factor of 5 . In addition, multipath effects are highly dynamic whereby the ionospheric ones are changing comparable slowly, what clarifies the necessity to analyze the specific given scenario to compensate ionospheric and multipath effects, like shown in Figure 5(d), instead of using statistical models. Here two different constellations are analyzed, both using the developed post-processing routine. The circles and the outside lines are indicating the positioning values and the .95 quantiles of a stand-alone GPS system in the given scenario, the inner lines and the crosses the corresponding values for a combination of GPS and Galileo. Thereby a virtual Galileo TLE datasets was implemented, using the already published Galileo constellation parameters [17]. Taking into account every effect with an impact of more than $1 \mathrm{~m}$ to the localization accuracy, it becomes possible to locate objects really 'lanespecific' for both constellation types in $95 \%$ of all cases, whereby the benefit of combining both systems is clearly visible when comparing the two .95 quantiles in Figure 5(d) in detail. Especially in terms of safety-critical services, like those in traffic systems, a guaranteed positioning of 'just' $95 \%$, referring to the outer circles in Figure 5(d) may not fulfill the necessary requirements. In contrast, the usage of both constellations at once, increase the lane-specific localization 
of up to $99.3 \%$ of all cases in the used scenario, without any mapping or pattern recognition techniques.

2) Impact to Lane-Specific Positioning: The motivating use case deals with a 'lane-specific' localization of traffic objects, which was introduced in Section II. Hence, upcoming localization failures in the driving direction would be acceptable as long as the corresponding traffic object can be localized lane-specific. Therefore Figure 6 visualizes the impact of the developed techniques to the mentioned use-case by just analyzing the amount of all positioning errors which are perpendicular to the driving direction, referring to the result figures the y-error. Again a virtual two-lane carriageway is

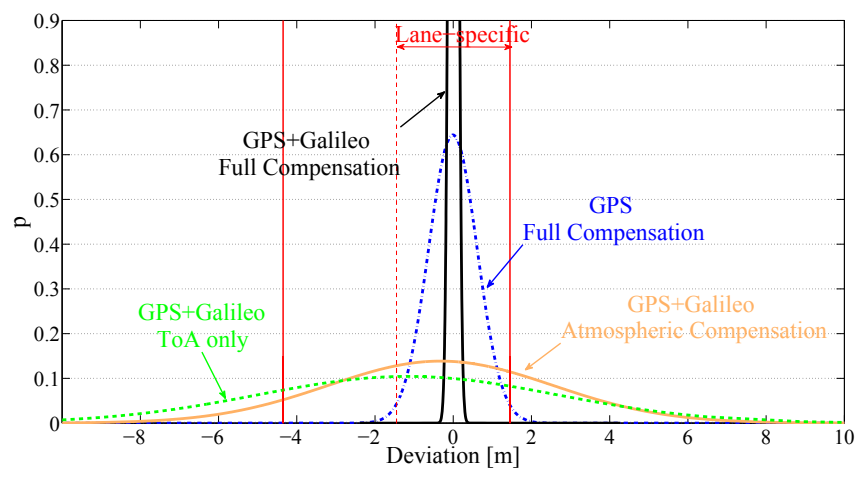

Fig. 6. Deviation perpendicular to the Driving Direction

used to visualize the performance. It is obvious, that both full compensated constellations are able to guarantee a failure probability below $5 \%$ each. Both error containing cases (with ionospheric and/or multipath effects) clearly display a high probabilty of 'fail-detections' concerning to the used lanespecific requirement.

\section{CONCLUSION}

This paper clarifies that the positioning accuracy of GPS is not sufficient for a lane-specific positioning of cars for the traffic prognosis, especially in urban scenarios, due to high multipath and ionospheric impact. Using the developed post-processing approach, the authors have identified certain accuracy enhancements in an exemplary urban scenario by using simulation knowledge to compensate the unpreventable residual error in satellite-based positioning techniques. In addition, the possible amount and the dynamic character of multipath and ionospheric influences to GNSS signals was analyzed, as well as the necessity to simulate these factor position-specific to enable this kind of post-processing techniques. In addition, the possible performance gain in using a combined GPS/Galileo constellation instead of using GPS as stand-alone system is highlighted.

The next steps will contain the implementation of the developed strategies within a Software-Defined GNSS receiver including additional strategies like data fusion mechanisms with real-time data from the vehicle's CAN bus. First experiments have already been successfully performed.

\section{ACKNOWLEDGMENT}

The work on this paper has been supported by Deutsche Forschungsgemeinschaft (DFG) within the Collaborative Research Center SFB 876 "Providing Information by ResourceConstrained Analysis", project B4.

\section{REFERENCES}

[1] B. Niehoefer, S. Subik and C. Wietfeld, Optimization Approach for Raytracing Analyses by Smart Minimization of the Modeling Level of Detail, 22nd IASTED International Symposia on Modelling and Simulation, Calgary, Canada, Jul 2011

[2] K. Nagel and C. Schreckenberg, A cellular automaton for freeway traffic, Journal de Physique I, No 12, p. 2221-2229. Dec. 1992.

[3] D. Weber et al.,OLSIM: Inter-Urban Traffic Information, Innovative Internet Community Systems, Lecture Notes in Computer Science, Springer Verlag, 2006.

[4] E. Lutz, A Marcov model for correlated land mobile satellite channels, International Journal of Satellite Communications, vol.. 14, p. 333-339, 1996.

[5] S. Scalise et al., Satellite channel impairments, Digital Satellite Communications, p. 503-528, Springer, 2010.

[6] B. Niehoefer, A. Lewandowski and C. Wietfeld, Evaluation of the Localization Accuracy of Satellite Systems for Traffic Flow Predictions, 24th International Technical Meeting of The Satellite Division of the Institute of Navigation (ION GNSS 2011), Portland, Sep 2011

[7] T.K. Sakar, Z. Ji and K. Kim and A. Medouri and M. Salazar-Palma, A Survey of Various Propagation Models for Mobile Communication, IEEE Antennas and Propagation Magazine, Vol. 45, No. 3, pages 51-82, Jun 2003.

[8] E. Lutz et al., The land mobile communication channel - recordings, statistics and channel model, IEEE Transaction on Vehicular Technology, p. 375-386, May 1991.

[9] W. Shen and L. Wynter, Real-time road traffic fusion and prediction with GPS and fixed-sensor data, 15th International Conference on Information Fusion (FUSION), pp. 1468-1475, San Bruno, USA, Jul 2012

[10] A. Amoroso, G. Marfia and M. Roccetti, GPS Position Errors in VANETS: Their Impact on a Real-World Accident Warning System, 1st AESS IEEE Conference on European Satellite Telecommunication, Rome, Italy, Oct 2012

[11] K. Rizk, Two-Dimensional Ray-Tracing Modeling for propagation Prediction in Microcellular Environments, IEEE Transactions on Vehiclular Technology, Vol. 46, No. 2, pages 508-518, May 1997

[12] B. Nava et al., Using NeQuick to Reconstruct the 3D Electron Density of the Ionosphere: Benefits and Capabilities in Single Frequency Positioning Applications, 1st AESS IEEE Conference on European Satellite Telecommunication, Rome, Italy, Oct 2012

[13] M. Schack., Comparison of path loss measurements and predictions at urban crossroads for C2C communications, Proceedings of the 5th European Conference on Antennas and Propagation (EUCAP),p. 28962900, Rome, Italy, April 2011.

[14] J. Gomez, A. Tayebi, F. M. Saez de Adana, and O. Gutierrez, Localization approach based on ray-tracing including the effect of human shadowing, Progress In Electromagnetics Research Letters, Vol. 15,pages $1-11,2010$.

[15] A. Lewandowski, R. Burda, S. Subik and C. Wietfeld, A Multiscale Simulation Environment for Performance Evaluation of high reliable heterogeneous Communication Networks, European Simulation and Modelling Conference (ESM), pages 131-136, Le Havre, France, October 2008

[16] F. Toran-Marto, J. Ventura-Traveset and J.C. de Mateo The ESA SISNET Project: Real-Time Access to the EGNOS Services across the Internet, 7th International Workshop on Digital Signal Processing Techniques for Space Communications,Lisbon, Portugal, Oct 2001.

[17] I. Benedicto., S.E. Dinwiddy, G. Gatti, K. Lucas and M. Lugert, GALILEO satellite system design and technology developments:, European Space Agency, Nov. 2000. 